\title{
POMEGRANATE MOLASSES AND RED GRAPE VINEGAR: CAN THEY ALLEVIATE DYSPLASTIC CHANGES IN CHEMICALLY INDUCED ORAL SQUAMOUS CELL CARCINOMA IN HAMSTERS?
}

\author{
Samah K. Ezzat *, Amira M Elsherbini * , Doaa AM Esmaeil ${ }^{* *}$ and Mohamed Abdulrahman ***
}

\begin{abstract}
Objective: Epithelial mesenchymal transformation (EMT) is an essential process for tumor progression. Targeting molecules in EMT might repress tumor progression. Pomegranate molasses and red grape vinegar are rich in polyphonic compounds with a significant therapeutic use. In this study we evaluated the possible protective effect of pomegranate molasses and red grape vinegar on dysplastic changes in chemically induced oral squamous cell carcinoma in hamsters.

Material and Methods: 76 male hamsters were divided equally into 4 groups (19 each). Group I served as control group with no intervention. Group II received topical application of $0.5 \%$ 7, 12-dimethylbenz[a]anthracene (DMBA) on the left buccal pouches for 16 weeks with no intervention. Group III received $0.5 \mathrm{ml}$ of Pomegranate molasses plus $0.5 \mathrm{ml}$ distilled water through ingestion daily which was concomitant with DMBA application and continued for 3 months post induction period. Group IV received a red grape vinegar in a protocol similar to group III. Then the pouches were collected, fixed and processed for H\&E, anti-TGF- $\beta$ and anti-CK-19 staining.

Results: Group II showed classical dysplastic changes with statistically significant higher expression of anti-TGF- $\beta$ and CK-19 ( $<<0.0001)$ compared to group III and IV. The later showed reduction of dysplastic changes and anti-TGF- $\beta$ and CK-19 expression. There was no significant difference between the two treatment modalities.

Conclusion: Both pomegranate molasses and red grape vinegar reduced the signs of dysplasia via reduction of TGF- $\beta$ and CK-19 expression and consequently reduction of the EMT. Both pomegranate molasses and red grape vinegar represent promising protective therapeutic modalities for reduction of dysplasia and carcinogenesis.
\end{abstract}

KEYWORDS: Pomegranate molasses, red grape vinegar, DMBA, TGF- $\beta$, CK-19

\footnotetext{
* Lecturer of Oral Biology, Faculty of Dentistry, Mansoura University, Egypt

** Lecturer of Oral Pathology, Faculty of Dentistry, Mansoura University, Egypt

*** Associate professor of Oral Biology, Faculty of Dentistry, Mansoura University, Egypt
} 


\section{INTRODUCTION}

It was estimated that most of deaths cases related to cancer could be diminished through changing the lifestyle, almost through dietary means ${ }^{(1,2)}$. Oral cancer is one of the common malignancies in the world ${ }^{(2,3)}$. It is a highly terrible disease that has become a serious public health concern. In 2018, cancers of the lip and oral cavity were collectively estimated at 354,864 new cases with deaths reaching 177,384 worldwide ${ }^{(4)}$. The prevalent oral cancer malignancy is oral squamous cell carcinoma (OSCC), that almost related to poor prognosis ${ }^{(5)}$.

Different systems of medicine in various countries have claimed and encouraged the use of edible substances, particularly those of plant origin, to manage diseases over last decades. This creates awareness of the possibility of natural agents to control cancer ${ }^{(6)}$. These agents are known as phytochemicals which were considered chemotherapeuticlchemo-preventive agents. Almost $47 \%$ of the market drugs that are used to treat cancers are derived from natural products or mimics ${ }^{(7)}$. The consumption of fruits and vegetables in diet daily found to reduce the incidence of cancer and its risk ${ }^{\left({ }^{8}\right)}$. In addition, great attention has been concentrated on foods of plant origin that is rich in polyphenols ${ }^{(6,9,10)}$. Huge number of various phytochemicals has been identified in vegetables and fruits that have enormous anticancer effects ${ }^{(11)}$. Phytochemicals are non-toxic substances and generally have a role in targeting many signaling pathways ${ }^{(10,12)}$.

A large number of clinical researches have announced the valuable effects of herbal medicines on the modulation of immunity, survival and goodness of life of patients suffering from cancer and in case of adopting a treatment system depending on a combination between the conventional anticancer agents together with the herbal medicines. Some countries all over the world such as China are being used the herbal medicines in combination with chemotherapy or radiotherapy to improve the effectiveness of cancer therapy and minimize the complications and side effects ${ }^{(13,14)}$.

Prescription medicine is not to be preferred today because of the high cost and their unwanted side effects such as effects of chemotherapy in cancer treatment ${ }^{(13)}$. These adverse effects may influence patients' quality of life and in some cases, it may be difficult to keep treatment with the conventional methods like radiotherapy or chemotherapy ${ }^{(15)}$. Studies were reported the anti-oxidative properties of the active components of herbal medicine. These compounds prevent lipid peroxidation, so they have anti-cancer properties ${ }^{(16)}$.

Though a large volume of worthy strategies has been created to minimize or manage these side effects, they are still deficient and an incomplete. Therefore, an alternate and efficient chemopreventive strategy to treat or prevent the side effects is required ${ }^{(17)}$.

The pomegranate molasses (PM) is a concentrated production manufactured by boiling of pomegranate juice without further adding any substances like sugar or other additives. Pomegranate molasses are still being produced by traditional methods. It is a thick, dark red liquid formed after cleaning, crushing, extraction, filtration, and evaporation in an open vessel or under vacuum, ${ }^{(18,19)}$ Additionally, the total phenolic contents of PM was reported to be nontoxic and four times greater than those found in the juice ${ }^{(20)}$.

The products of Grape such as vinegar, wine and juice contain a large amount of flavonoids, which have considerable biological activities including antioxidant effects, (21). Cardiovascular benefits and cancer chemo-preventive activity have been suggested to be due to the flavonoid antioxidant effect of grape ${ }^{(22)}$. Many in vitro studies and experimental models in addition to human trials have been investigated the antioxidant potential of grapes. They yielded positive results with highlighting on the correlation between the investigated biomarkers and the disease prevention ${ }^{(23)}$. 
The transforming growth factor $\beta$ (TGF $\beta$ ) has implicated in epithelial mesenchymal transition (EMT) ${ }^{(24,25)}$. Many growth factors can be expressed by the effect of TGF $\beta$. This cause further reproduction in the signaling activities of these growth factors ${ }^{25,26)}$. During carcinogenesis, there is an excess of signaling pathways that induce the process of epithelial mesenchymal transitions. In this state, the TGF $\beta$ play a significant role in EMT. The transforming growth factor $\beta$ family includes multiple polypeptides with different physiological and developmental roles ${ }^{(27)}$. Mature TGF $\beta$ ligands are created when different types of cells are activated in the tissue environment, and on secretion of specific proteases and later expression of receptors of integrin, they perform the chemical cleavage and conformational distortion of the latent ligands ${ }^{(28)}$.

Extracellular matrix (ECM) changes characteristically during cancer progression ${ }^{(29)}$. TGF $\beta$ secreted by tumor cells is able to induce the synthesis of new ECM and remodeling ECM based on metalloproteases secretion and phenotypic changes that induce cell invasion (30). In addition to cell-cell contact remodeling during epithelial mesenchymal interaction, transforming growth factor $\beta$ induce integrin receptor-ECM remodeling that facilitates motility of cancer cell. Certain integrins are newly synthesized during EMT and other integrins are downregulated ${ }^{(31)}$.

Cytokeratins (CKs) form the largest intermediate filament protein and act a multigene family with more than 20 different types of polypeptides that are divided into acidic type I and basic type II. Normal epithelial cells express at least one type of acidic keratin (CK9-CK20) and one of type of basic keratins (CK1-CK8). CKs form the epithelial cells cytoskeleton, and their main functions is to maintain the integrity of epithelial cell, have roles in cell signaling, stress responses, and apoptosis ${ }^{(32)}$.

CK19 is considered one of the three main keratins besides CK8 and CK18. It is expressed in simple or stratified epithelium and in various carcinomas ${ }^{(33)}$. The release of intact, nondegraded CK molecules has not yet been demonstrated. Interestingly, CK19 expression is the most common single marker used for the RT-PCR-based detection of disseminated tumor cells (DTCs) of some types of cancers as breast cancer patients, and the detection of DTCs expressing of this particular cytokeratin is correlated to an unfavorable prognosis because of metastatic relapse ${ }^{(34)}$. It can be supposed that CK19 not only is a marker for cells of epithelial tumor but also may have some biologically pertinent functions in early metastatic spread ${ }^{(35)}$.

Therefore, and according to the above-mentioned data, the present study was conducted to detect the chemo-preventive effects of pomegranate molasses and red grape vingar in the 7,12-dimethylbenz[a] anthracene (DMBA)-induced hamster cheek pouch model. A long-term experiment (16-weeks topical DMBA) coincident with the ingestion of pomegranate molasses and red grape vinegar was done to monitor their effects on cancer induction or prevention in hamster cheek pouch.

\section{Methodology:}

\section{Animals' preparation and experimental approach}

\section{Sample size:}

Seventy-six pathogen free male golden Syrian hamsters were selected for this study. They were 6 weeks old and weighed 150-200 g. The sample size was determined using $\mathrm{G}^{*}$ Power software (version 3.1.9.4 Universitat Dusseldorf Germany). The family test was f test, the statistical test was oneway ANOVA, and type of power analysis was: A priori: compute required sample size-given alpha, power, and effect size. With input parameters: effect size/0.40, $\alpha$ error probability/0.05, and power/0.80. The calculated sample size was 76 . 


\section{DMBA application and tumor induction protocol}

Left buccal pouches of hamsters were topically treated with $0.5 \%$ 7, 12-dimethylbenz[a]anthracene (DMBA) (Sigma Chemical Company, St Louis, MO, USA) dissolved in 100 ul corn oil at appropriate concentrations for topical application (Acros organics, New Jersy, USA) with a paintbrush three times/week for 16 weeks (36).

\section{Grouping:}

For acclimatization, rats were kept for one week before the onset of experiment and housed in polycarbonate cages at room temperature $25^{\circ} \mathrm{C}$, 12 hours light dark cycle in the research laboratory of the Nile Center for Experimental Research, Mansoura, Egypt. Water and food were allowed to hamsters ad libitum. All animals appeared healthy before the study. The experiments were carried out according to the protocol approved by the local committee of ethics, Faculty of Dentistry, University of Mansoura.

The hamsters were randomly divided into four equal groups, (19 hamsters each). The animals of group I received no treatment, while group II had a topical DMBA application on the buccal pouch for 16 weeks. Group III received pomegranate molasses treatment through ingestion using gavage tube with a dose of $0.5 \mathrm{ml}(\mathrm{PM})$ plus $0.5 \mathrm{ml}$ distilled water (24) daily concomitant with cancer induction using DMBA application on the buccal pouches for 16 weeks and pomegranate molasses ingestion continued for 3 months post induction period. Group IV received a red grape vinegar in a protocol similar to group III, during induction period and post induction period with a dose and delivery method similar to group III. Pomegranate molasses and red grape vinegar were purchased from the local market in Mansoura, Egypt.

\section{Biopsy collection}

The animals were sacrificed at the end of the experiment, 6 hours after the last treatment. The cheek pouch was harvested, being fixed in $10 \%$ PBS-buffered formalin for histopathology ${ }^{(37)}$. The sections stained with HE stains as a routine staining. Then, immunohistochemical staining for TGF- $\beta 1$ and cytokeratin 19 rabbit monoclonal antibodies was performed.

\section{Image and statistical analysis:}

Ten fields were examined using thresholding technique to measure the intensity of positive immunostaining reaction in all groups. Data were coded, tabulated using Statistical Package for Social Science software computer program version 26 (SPSS, Inc., Chicago, IL, USA). The data were tested for normality using Shapiro Wick test. Quantitative parametric data were presented in mean and standard deviation (SD). One-way analysis of variance (ANOVA) followed by posthoc Tukey tests were used. $\mathrm{P}$ value less than 0.05 was considered statistically significant.

\section{RESULTS}

\section{H\&E stain}

H\&E sections of group I showed thin keratinized stratified squamous epithelium, with basal cells arranged in a palisading manner with normal cell density and cytological features of nuclei. A normal distribution and arrangement of epithelial layers could be seen. The basal cells rested on intact and flat basement membrane. The underlying connective tissue revealed collagen fibers with fibroblasts, (fig 1

A). Group II revealed prominent signs of dysplasia, as hyperplasia, elongated deeply invading drop shaped rete processes (a), marked basal hyperplasia (b) with loss of polarity, hyperchromatic and pleomorphic nuclei. Basement membrane showed discontinuity and underling lamina propria was invaded with epithelial cells either scattered or in the form of cell nests (c). Irregular epithelial stratification, premature keratinization in lower third of epithelium (d), (fig 1 B). While groups III and IV showed marked regression in signs of dysplasia. In group III, discontinuity of basement membrane (e) and some cellular and nuclear pleomorphism could be seen (fig $1 \mathrm{C}$ ). Disordered maturation from basal to top cells, thin extensions (nodules) of basal cells 
inside C.T in a strand form (f) appeared in group IV (Fig. 1 D).

\section{Immunohistochemical results:}

The immunohistochemical positive reaction of TGF- $\beta$ sections was detected as brown deposit at the cytoplasm of epithelial cells and fibroblasts. Oneway ANOVA test for TGF- $\beta$ revealed significant difference between group II and both group III and IV $(\mathrm{p}<0.05)$ (Table 1). Group I showed mild expression of TGF- $\beta$, while the strongest expression was noticed in Group II. However, groups III and IV revealed decreased expression in comparison to Group II (Fig. 2). CK 19 immunohistochemical positive reaction was detected in cytoplasm of epithelial cells, with the least positive reaction at group I, while the most intense reaction is in group II $(\mathrm{p}<0.05)$, where groups III and IV expressed moderate reaction, (Fig. 3). There was no statistically significant difference between the two modalities (III, IV) regarding both TGF- $\beta$ and CK 19 where $\mathrm{p}$ value $=0.6$ and 0.9 respectively.
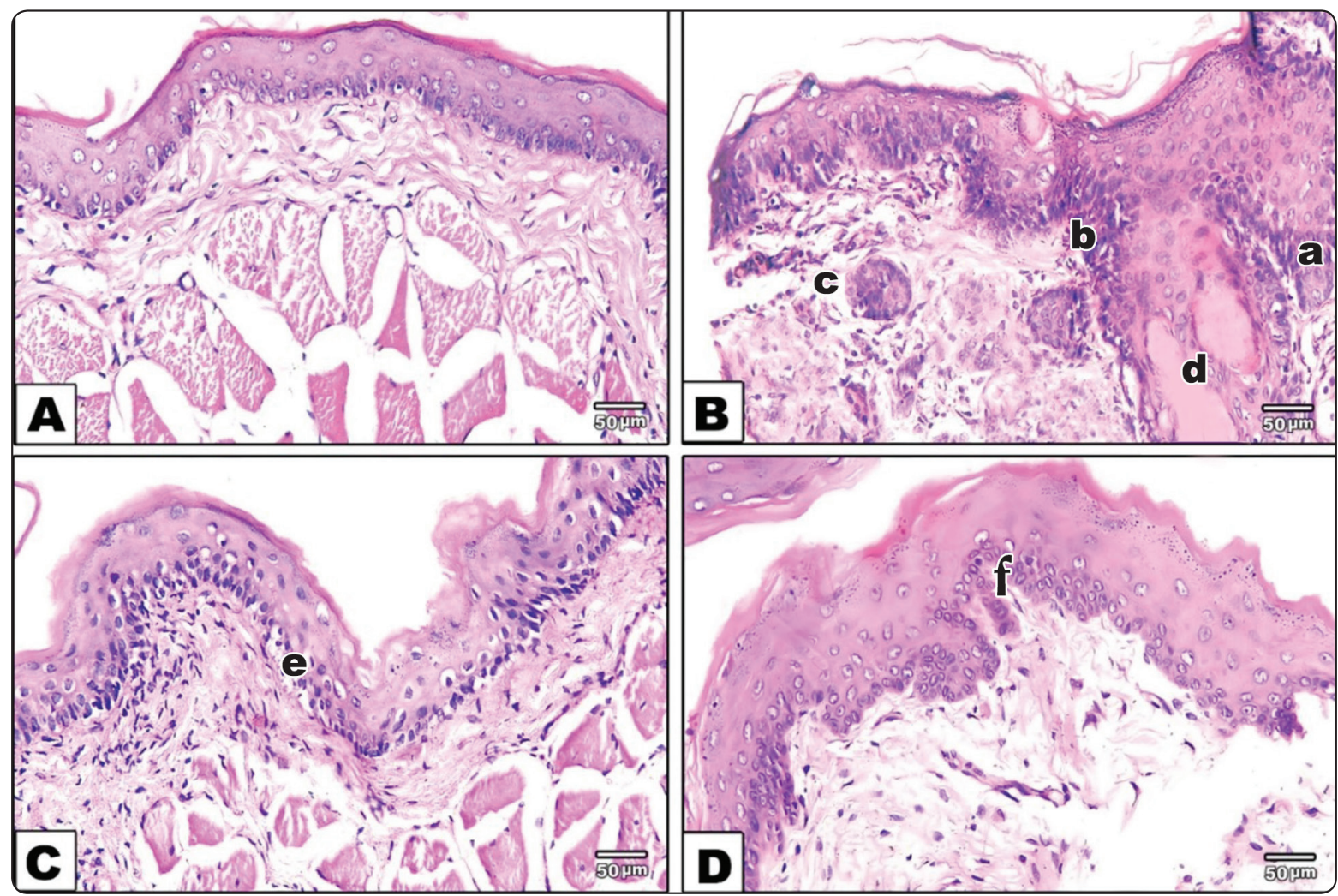

Fig. (1) Photomicrograph showing, H\&E-stained section of buccal pouches of hamsters for groups I, II, III and IV (H\&E 200 x).

TABLE (1): Image analysis of TGF- $\beta$ and CK-19 expression results:

\begin{tabular}{|c|c|c|c|c|c|c|}
\hline Parameter & Group I & Group II & Group III & Group IV & F value & P value \\
\hline TGF- $\beta$ & $0.76 \pm 0.62^{\mathrm{c}}$ & $11.15 \pm 3.17^{\mathrm{b}}$ & $5.76 \pm 1.36^{\mathrm{a}}$ & $5.07 \pm 1.08^{\mathrm{a}}$ & 102.35 & 0.0001 \\
\hline CK-19 & $0.78 \pm .68^{\mathrm{c}}$ & $10.87 \pm 2.12^{\mathrm{b}}$ & $4.37 \pm 1.62^{\mathrm{a}}$ & $4.29 \pm 1.86^{\mathrm{a}}$ & 122.01 & 0.0005 \\
\hline
\end{tabular}

Data were expressed in mean $\pm S D$, using One Way ANOVA test followed by Tukey post-hoc test. p value $<0.05$ was considered significant. Different letters are assigned to statistically significant different groups. 

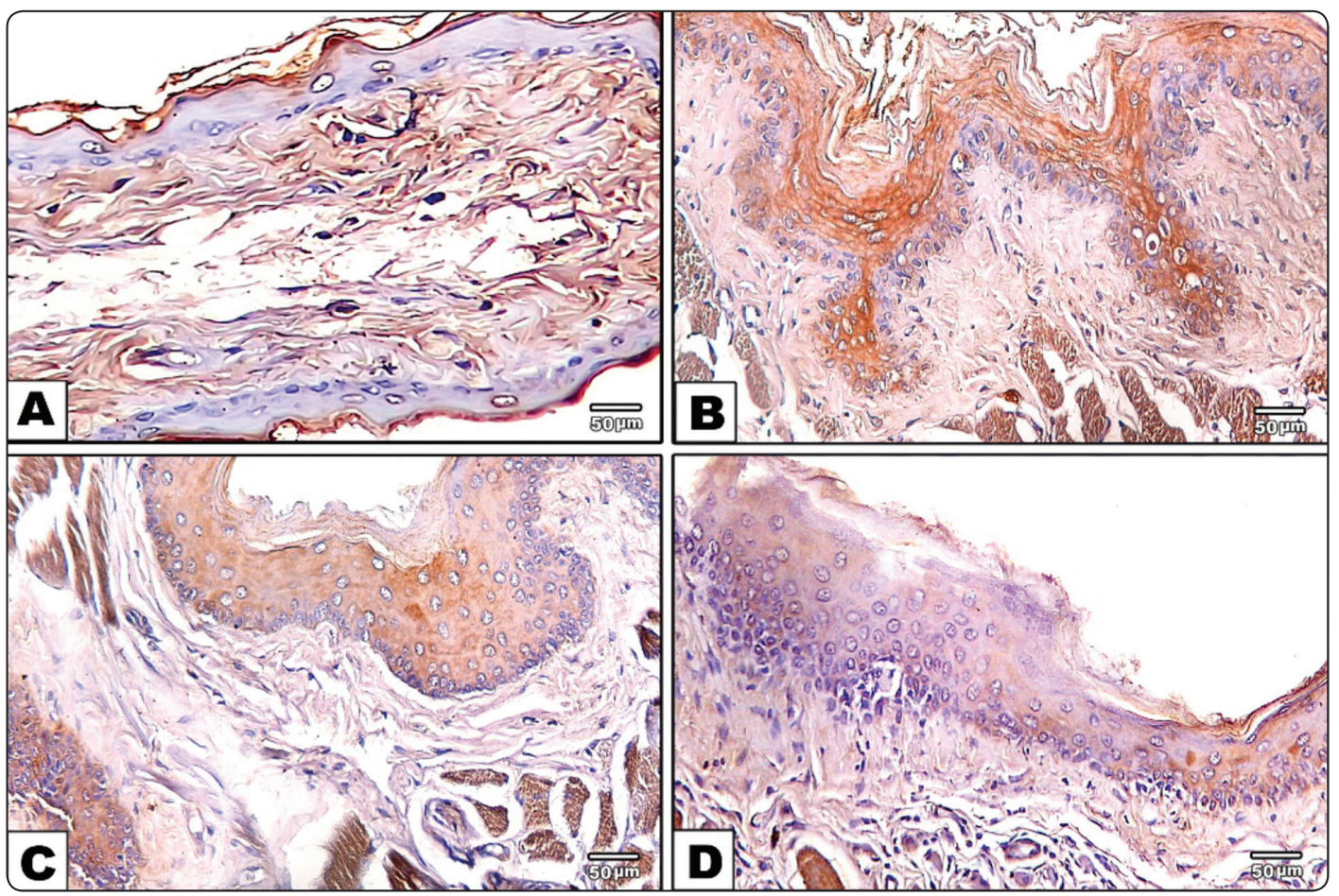

Fig. (2) Photomicrograph showing varying expressions of TGF-B, where group I showed weak expression, $(\%$ of reaction area $=$ 0.552), (A). Group II: intense reaction of epithelium which extends to the invasive epithelium into C.T, (\% of reaction area $=12.427)$, (B). Group III showing mild to moderate reaction (\% of reaction area $=5.674),(\mathrm{C})$. Group IV: showing mild to moderate reaction (\% of reaction area $=3.98)$, (D). (IHC $200 \mathrm{x}$ )

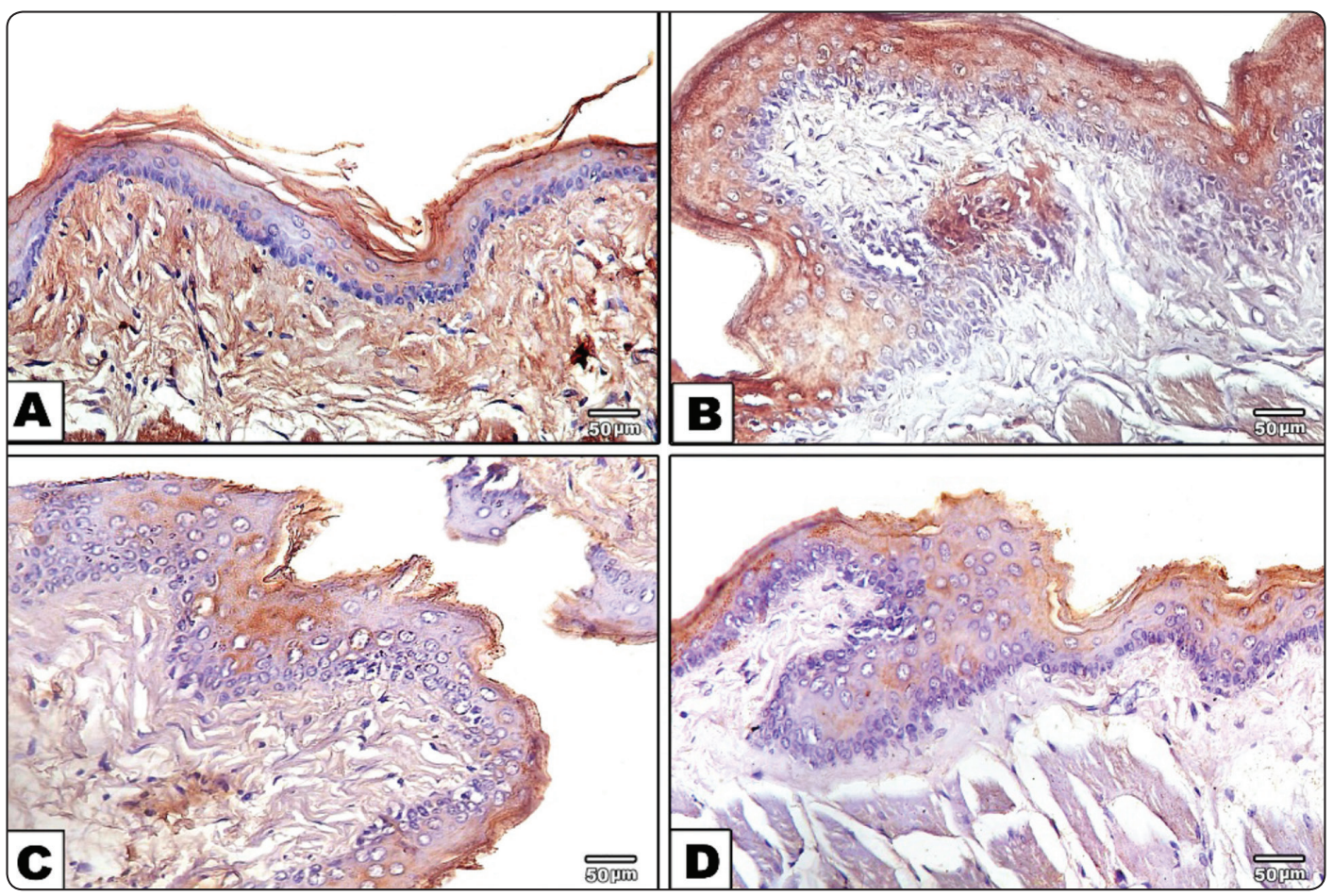

Fig. (3) Photomicrograph showing varying expression of Cytokeratin 19, where group I showed weak expression, (\% of reaction area $=0.78),(\mathrm{A})$. Group II: intense reaction of epithelium which extends to the invasive epithelium into C.T, (\% of reaction area $=11.16),(B)$. Group III showing mild to moderate reaction $(\%$ of reaction area $=3.674),(\mathrm{C})$. Group IV: showing mild to moderate reaction ( $\%$ of reaction area $=3.22$ ), (D). (IHC $200 \mathrm{x}$ ) 


\section{DISCUSSION}

The efficacy of herbal medicine has drawn attention throughout recent years to detect its effect on cancer prevention. This is due to its decreased cost, ease of extraction and less adverse effects on body tissues in contrast to other chemotherapeutic agents. Several plant products have displayed very hopeful anti-tumor characteristics in vitro, however, they need to be more assessed in humans (38). Much researches have revealed that the products of natural origin show a wide range of biologic activities such as immune system activation, antibacterial, antiviral, anti-inflammatory, antioxidant, anti-mutagenic and anti-cancer effects ${ }^{(39)}$.

In the present study, we selected two products of plant origin to verify their effects in prevention of oral tumor when given simultaneously and after induction of oral squamous cell carcinoma (SCC) in hamsters. Hamsters were selected as a model for SCC induction. Pomegranate molasses and red grape vinegar are fruits products, available in the market and easily applied and given to the animal experimentally.

Epithelial-to-mesenchymal transition (EMT) is a tightly self-regulated process implicated in three main steps, embryogenesis, adult tissue repair and cancer progression. During EMT, the epithelial cells acquire the properties of mesenchymal cells via several genetic and molecular pathways. Worth mentioning, the (TGF- $\beta$ )/Smads pathway has been reported to be the strongest EMT inducer ${ }^{(40,41)}$.

TGF-ßs are proinflammatory cytokines, that might act as a cancer promotor or repressor according to the stage of carcinogenesis. They either function directly through Smads pathway or indirectly via upregulation of EMT-related transcription factors, regulating distinct processes, such as cytoskeleton organization, cell growth, survival, migration or invasion ${ }^{(42,43)}$. TGF- $\beta$ can further induce important nuclear transcriptional factors that activate $\mathrm{Cy}$ tokeratin19 promoter. Cytokeratin 19 is a progenitor cell marker and tumor stem cell marker, that play an important role in EMT and cancer progression ${ }^{(44)}$.
H\&E results of our study revealed group I cheek pouch mucosa with normal epithelial thickness and normal distribution of its layers and nearly flat basement membrane. Submucosa was formed of loose delicate tissue. While group II sections showed sever dysplastic changes as basilar hyperplasia and deeply invasive rete processes. These findings are in agreement with Selvasundaram et al 2002, who stated that DMBA caused dysplasia and hyperplasia in all the pouches of animals subjected to it ${ }^{(32)}$. In addition, the appearance of hyperchromatic nuclei and deeply invaded rete processes in group II agrees with the occurrence of hyperproliferation in hamster cheek pouch after 3 weeks application of DMBA as shown by increased BrdU-labeling index in the study done by Xin et al 2002, ${ }^{(33)}$.

Systemic administration of pomegranate molasses and red grape vinegar at the induction and post-induction stages led to a decrease in the signs of dysplasia under the microscope and reduced the size and number of DMBA-induced visible tumors.

Groups III and IV sections manifested marked decrease in the signs of dysplasia and minimal invasion of epithelium into underlying connective tissue. These results were consistent with Mandal et al 2017 who reported that pomegranate extract and seed oil decreased incidence of DMBA induced breast cancer in rats. This may be due to the pomegranate ability and its extracts to hinder DMBA- mediated inflammatory signaling cascades via decreasing COX-2, nitric oxide, IL-6, and NF$\mathrm{kb}$ expression ${ }^{(3)}$.

A study performed by George et al 2011, proved that fruit extract of pomegranate (PFE) and diallyl sulfide (DAS) as chemo-preventive agents alone and in combination reduced the degree of dysplastic changes in carcinoma. PFE alone delayed tumor incidence and onset by 55\%, while in combination of PFE+DAS at low doses, they synergistically decreased tumor incidence more potentially (84\%). This was attributed to ability of pomegranate extracts to inhibit the expression of phosphorylated 
ERK1/2, JNK1 and activated NF- $x$ B/p65, IKK $\alpha$, I $x \mathrm{~B} \alpha$ phosphorylation ${ }^{(34)}$.

Scientific evidence suggested that the therapeutic effects of pomegranate molasses might be related to the anti-oxidative characteristics of its polyphenolic compounds such as phytochemicals ${ }^{(14)}$. Where it showed a great role in the prevention of prostate cancer, oxidation prevention of high-density and low-density lipoprotein and the stimulation of T-cell functions and production of cytokines ${ }^{(35)}$.

The marked regression in the signs of dysplasia observed in group VI may be due to antioxidant activity of red grape vinegar $(\mathrm{RGV})$ through the action of its phenolic compounds. The different degrees in the improvement state in group IV's sections may be related to the variation in the expression of the oncogene when cancer cells exposed to antioxidant (phenols from RGV). Thus, the antioxidative action of red grape vinegar is suppressed carcinogenesis by preventing oxidative damage to DNA, ${ }^{(36)}$. Also, grape antioxidants produce anti-inflammatory effect by its anti COX effect, ${ }^{(37)}$. Furthermore, the antioxidant effect of grape has anticancer properties partially related to their immune-potentiating activities through the enhancements of lymphocyte proliferation, cytotoxicity of NK cell, CD4+/ CD8+ ratio, IL-2, and IFN- $\gamma$ productions. All these interpret the reduction in signs of dysplasia seen in group IV sections ${ }^{(38)}$.

The immunohistochemical results of group III with marked decrease in percentage of reaction of both markers in comparison to group II could be easily detected. Pomegranate extract showed considerable pro-apoptotic and anti-proliferative activity against highly aggressive human PC-3 cells ${ }^{(45)}$. Previous research has reported that pomegranate with its phytochemical constituents can efficiently affect multiple signaling pathways involved in inflammation, cellular transformation, hyperproliferation, angiogenesis, initiation of tumorigenesis, and eventually suppressing the final steps of tumorigenesis and metastasis ${ }^{(10,46)}$. So, the absence of epithelial invasion into the C.T in group III may be due to the ability of pomegranate constituents to modulate transcription factors, modulate molecules that regulate cell cycle, molecules of cell adhesion, pro-inflammatory mediators, and growth factors in various cancers ${ }^{(47)}$.

Meanwhile, the protective effect of RGV may be due to the diverse mixture of biologically active compounds including gallic acid, catechin, epicatechin, chlorogenic acid, caffeic acid, syringic acid, ferulic acid. In addition to synergistic action of syringic acid, melatonin, and resveratrol. Syringic acids were reported to reduce the process of carcinogenesis in oral mucosa through enhancing cell surface glycol-conjugation and cytokeratin expression modification ${ }^{(36,46)}$. This effect may be due to induction of cell cycle arrest and apoptosis in cancer cells ${ }^{(48)}$, and the prevention of carcinogenesis and cancer progression in rodent models ${ }^{(49)}$.

This observation may interpret the regression in signs of dysplasia in histological sections of group IV and the decrease in the percentage of TGF $\beta$ and cytokeratin 19 immunostains in group III and IV compared to both group I and group II. This may be attributed to the ability of both PM and RGV to reduce TGF- $\beta$ and CK-19 expression and consequently reduce the EMT.

\section{CONCLUSION}

Based on the results of the current study, pomegranate molasses and red grape vinegar caused significant regression of oral cancer progression as they exert anti-inflammatory and anti-proliferative effects potentiating their chemo-preventive impacts. Therefore, additional in vitro and in vivo studies are needed to evaluate the combined effect of pomegranate molasses and red grape vinegar together or with other agents. In addition, welldesigned human clinical trials are essential to evaluate their usefulness. Further studies are needed to explore the molecular mechanisms of their tumor suppressive effect at this level. 


\section{REFERENCES}

1. Key TJ, Schatzkin A, Willett WC, Allen NE, Spencer EA, Travis RC. Diet, nutrition and the prevention of cancer. Public health nutrition. 2004;7(1a):187-200.

2. Barnard RJ. Prevention of Cancer Through Lifestyle Changes. Evidence-based complementary and alternative medicine : eCAM. 2004;1(3):233-9.

3. Ghantous Y, Abu Elnaaj I. Global incidence and risk factors of oral cancer. Harefuah. 2017;156(10):645-9.

4. Bray F, Ferlay J, Soerjomataram I, Siegel RL, Torre LA, Jemal A. Global cancer statistics 2018: GLOBOCAN estimates of incidence and mortality worldwide for 36 cancers in 185 countries. CA: a cancer journal for clinicians. 2018;68(6):394-424.

5. Torres-Rendon A, Roy S, Craig GT, Speight PM. Expression of $\mathrm{Mcm} 2$, geminin and Ki67 in normal oral mucosa, oral epithelial dysplasias and their corresponding squamous-cell carcinomas. British journal of cancer. 2009;100(7):1128-34.

6. Gundgaard J, Nielsen JN, Olsen J, Sørensen J. Increased intake of fruit and vegetables: estimation of impact in terms of life expectancy and healthcare costs. Public health nutrition. 2003;6(1):25-30.

7. Newman DJ, Cragg GM. Natural products as sources of new drugs over the last 25 years. Journal of natural products. 2007;70(3):461-77.

8. Boeing H, Bechthold A, Bub A, Ellinger S, Haller D, Kroke A, et al. Critical review: vegetables and fruit in the prevention of chronic diseases. European journal of nutrition. 2012;51(6):637-63.

9. Huang WY, Cai YZ, Zhang Y. Natural phenolic compounds from medicinal herbs and dietary plants: potential use for cancer prevention. Nutrition and cancer. 2010;62(1):1-20.

10. Khan N, Afaq F, Mukhtar H. Cancer chemoprevention through dietary antioxidants: progress and promise. Antioxidants \& redox signaling. 2008;10(3):475-510.

11. Anand P, Kunnumakkara AB, Sundaram C, Harikumar KB, Tharakan ST, Lai OS, et al. Cancer is a preventable disease that requires major lifestyle changes. Pharmaceutical research. 2008;25(9):2097-116.

12. DiMarco-Crook C, Xiao H. Diet-based strategies for cancer chemoprevention: the role of combination regimens using dietary bioactive components. Annual review of food science and technology. 2015;6:505-26.

13. Yin SY, Wei WC, Jian FY, Yang NS. Therapeutic applications of herbal medicines for cancer patients. Evidence- based complementary and alternative medicine: eCAM. 2013;2013:302426.

14. Ohnishi S, Takeda H. Herbal medicines for the treatment of cancer chemotherapy-induced side effects. 2015;6(14).

15. Akin S, Can G, Aydiner A, Ozdilli K, Durna Z. Quality of life, symptom experience and distress of lung cancer patients undergoing chemotherapy. European journal of oncology nursing : the official journal of European Oncology Nursing Society. 2010;14(5):400-9.

16. Ahmed MA, El Morsy EM, Ahmed AA. Pomegranate extract protects against cerebral ischemia/reperfusion injury and preserves brain DNA integrity in rats. Life sciences. 2014;110(2):61-9.

17. Schirrmacher V. From chemotherapy to biological therapy: A review of novel concepts to reduce the side effects of systemic cancer treatment (Review). Int J Oncol. 2019;54(2):407-19.

18. Poyrazoğlu E, Gökmen V, Artik N. Organic Acids and Phenolic Compounds in Pomegranates (Punica granatum L.) Grown in Turkey. Journal of Food Composition and Analysis. 2002;15:567-75.

19. Kaya A, Sozer N. Rheological behaviour of sour pomegranate juice concentrates (Punica granatum L.). International Journal of Food Science \& Technology. 2005;40:223-7.

20. Chalfoun-Mounayar A NR, Yared P, Khairallah S., Chahine R. Antioxidant and Weight Loss Effects of Pomegranate Molasses. Journal of Applied Pharmaceutical Science 2012;02 (06): 45-50.

21. PezzutoJM.Grapes and human health: aperspective.Journal of agricultural and food chemistry. 2008;56(16):6777-84.

22. Iriti M, Faoro F. Bioactivity of grape chemicals for human health. Natural product communications. 2009;4(5):611-34.

23. Castilla P, Echarri R, Dávalos A, Cerrato F, Ortega H, Teruel JL, et al. Concentrated red grape juice exerts antioxidant, hypolipidemic, and antiinflammatory effects in both hemodialysis patients and healthy subjects. The American journal of clinical nutrition. 2006;84(1):252-62.

24. Moustakas A, Heldin CH. Signaling networks guiding epithelial-mesenchymal transitions during embryogenesis and cancer progression. Cancer science. 2007;98(10):1512-20.

25. Derynck R, Muthusamy BP, Saeteurn KY. Signaling pathway cooperation in TGF- $\beta$-induced epithelial-mesenchymal transition. Current opinion in cell biology. 2014;31:56-66.

26. Moustakas A, Heldin CH. Induction of epithelial-mesenchymal transition by transforming growth factor $\beta$. Seminars in cancer biology. 2012;22(5-6):446-54. 
27. Moustakas A, Heldin CH. The regulation of TGFbeta signal transduction. Development (Cambridge, England). 2009;136(22):3699-714

28. Travis MA, Sheppard D. TGF- $\beta$ activation and function in immunity. Annual review of immunology. 2014;32:51-82.

29. . !!! INVALID CITATION !!! \{\}.

30. Tsubakihara Y, Moustakas A. Epithelial-Mesenchymal Transition and Metastasis under the Control of Transforming Growth Factor $\beta$. International journal of molecular sciences. 2018;19(11).

31. Parvani JG, Galliher-Beckley AJ, Schiemann BJ, Schiemann WP. Targeted inactivation of $\beta 1$ integrin induces $\beta 3$ integrin switching, which drives breast cancer metastasis by TGF- $\beta$. Molecular biology of the cell. 2013;24(21):3449-59.

32. Coulombe PA, Omary MB. 'Hard' and 'soft' principles defining the structure, function and regulation of keratin intermediate filaments. Current opinion in cell biology. 2002;14(1):110-22.

33. Chu PG, Weiss LM. Keratin expression in human tissues and neoplasms. Histopathology. 2002;40(5):403-39.

34. Pantel K, Brakenhoff RH, Brandt B. Detection, clinical relevance and specific biological properties of disseminating tumour cells. Nature reviews Cancer. 2008;8(5):329-40.

35. Alix-Panabières C, Vendrell JP, Slijper M, Pellé O, Barbotte E, Mercier G, et al. Full-length cytokeratin-19 is released by human tumor cells: a potential role in metastatic progression of breast cancer. Breast cancer research : BCR. 2009;11(3):R39.

36. Guan XB, Sun Z, Chen XX, Wu HR, Zhang XY. Inhibitory effects of Zengshengping fractions on DMBA-induced buccal pouch carcinogenesis in hamsters. Chinese medical journal. 2012;125(2):332-7.

37. Sun Z, Sood S, Li N, Yang P, Newman RA, Yang CS, et al. Chemoprevention of 7,12-dimethylbenz[a]anthracene (DMBA)-induced oral carcinogenesis in hamster cheek pouch by topical application of a dual inhibitor of epidermal growth factor receptor (EGFR) and ErbB2 tyrosine kinases. Oral oncology. 2008;44(7):652-7.

38. Desai AG, Qazi GN, Ganju RK, El-Tamer M, Singh J, Sax- ena AK, et al. Medicinal plants and cancer chemoprevention. Curr Drug Metab. 2008;9(7):581-91.

39. Espín JC, García-Conesa MT, Tomás-Barberán FA. Nutraceuticals: facts and fiction. Phytochemistry. 2007;68(2224):2986-3008.

40. Xu J, Lamouille S, Derynck R. TGF-beta-induced epithelial to mesenchymal transition. Cell Res. 2009;19(2):156-72.

41. Ribatti D, Tamma R, Annese T. Epithelial-Mesenchymal Transition in Cancer: A Historical Overview. Transl Oncol. 2020;13(6):100773-.

42. Essa AAM, Deraz EM, Metwaly HA. Enhanced expression of transforming growth factor beta one (tgf- $\beta 1)$ during progression of oral epithelial dysplasia to carcinoma. . J Egyptian Dental Journal. 2016;62(Issue 1 - January (Part 3)):739-46

43. Valcourt U, Kowanetz M, Niimi H, Heldin CH, Moustakas A. TGF-beta and the Smad signaling pathway support transcriptomic reprogramming during epithelial-mesenchymal cell transition. Molecular biology of the cell. 2005;16(4):1987-2002.

44. Zhuo J-Y, Lu D, Tan W-Y, Zheng S-S, Shen Y-Q, Xu X. CK19-positive Hepatocellular Carcinoma is a Characteristic Subtype. Journal of Cancer. 2020;11(17):5069-77.

45. Malik A, Afaq F, Sarfaraz S, Adhami VM, Syed DN, Mukhtar H. Pomegranate fruit juice for chemoprevention and chemotherapy of prostate cancer. Proceedings of the National Academy of Sciences of the United States of America. 2005;102(41):14813-8.

46. Faria A, Calhau C. The bioactivity of pomegranate: impact on health and disease. Critical reviews in food science and nutrition. 2011;51(7):626-34.

47. Sharma P, McClees SF, Afaq F. Pomegranate for Prevention and Treatment of Cancer: An Update. Molecules (Basel, Switzerland). 2017;22(1).

48. Aggarwal BB, Bhardwaj A, Aggarwal RS, Seeram NP, Shishodia S, Takada Y. Role of resveratrol in prevention and therapy of cancer: preclinical and clinical studies. Anticancer research. 2004;24(5a):2783-840.

49. Garvin S, Ollinger K, Dabrosin C. Resveratrol induces apoptosis and inhibits angiogenesis in human breast cancer xenografts in vivo. Cancer letters. 2006;231(1):113-22. 